\title{
Deuterated $\mathrm{H}_{3}^{+}$in proto-planetary disks
}

\author{
C. Ceccarelli ${ }^{1}$ and C. Dominik ${ }^{2}$ \\ ${ }^{1}$ Laboratoire d'Astrophysique, Observatoire de Grenoble, BP 53, 38041 Grenoble Cedex 09, France \\ e-mail: cecilia.ceccarelli@obs.ujf-grenoble.fr \\ 2 Sterrenkundig Instituut "Anton Pannekoek", Kruislaan 403, 1098SJ Amsterdam, The Netherlands
}

Received 4 March 2005 / Accepted 6 June 2005

\begin{abstract}
Probing the gas and dust in proto-planetary disks is central for understanding the process of planet formation. In disks surrounding solar type protostars, the bulk of the disk mass resides in the outer midplane, which is cold $(\leq 20 \mathrm{~K})$, dense $\left(\geq 10^{7} \mathrm{~cm}^{-3}\right)$ and depleted of CO. Observing the disk midplane has proved, therefore, to be a formidable challenge. Ceccarelli et al. (2004) detected $\mathrm{H}_{2} \mathrm{D}^{+}$emission in a proto-planetary disk and claimed that it probes the midplane gas. Indeed, since all heavy-elements bearing molecules condense out onto the grain mantles, the most abundant ions in the disk midplane are predicted to be $\mathrm{H}_{3}^{+}$and its isotopomers. In this article, we carry out a theoretical study of the chemical structure of the outer midplane of proto-planetary disks. Using a self-consistent physical model for the flaring disk structure, we compute the abundances of $\mathrm{H}_{3}^{+}$and its deuterated forms across the disk midplane. We also provide the average column densities across the disk of $\mathrm{H}_{3}^{+}, \mathrm{H}_{2} \mathrm{D}^{+}, \mathrm{HD}_{2}^{+}$and $\mathrm{D}_{3}^{+}$, and line intensities of the ground transitions of the ortho and para forms of $\mathrm{H}_{2} \mathrm{D}^{+}$and $\mathrm{HD}_{2}^{+}$respectively. We discuss how the results depend on the cosmic ray ionization rate, dust-to-gas ratio and average grain radius, and general stellar/disk parameters. An important factor is the poorly understood freeze-out of $\mathrm{N}_{2}$ molecules onto grains, which we investigate in depth. We finally summarize the diagnostic values of observations of the $\mathrm{H}_{3}^{+}$isotopomers.
\end{abstract}

Key words. ISM: abundances - ISM: molecules - stars: formation - astrochemistry - planetary systems: protoplanetary disks

\section{Introduction}

Proto-Planetary disks are the sites of planet formation. Their physical, dynamical and chemical structure and evolution determine if, when, how, where and what planets form. Two very important parameters that are difficult to determine are the gas mass of the disk, and the ionization degree. Critical questions that need to be answered by observations are: what is the evolution of the gaseous component of the disk, in particular with respect to the dusty component? Is it dispersed before or after dust coagulates into planetesimals and/or rocky planets? At what radius? By what process? At the same time, theory predicts that the accretion in the disk is regulated by its ionization degree (Balbus \& Hawley 1998; Gammie 1996). So the questions here are: what is actually the measured ionization degree across the disk? What ionizes the gas? Cosmic rays and/or X-rays? Where do the two effects balance each other, if they do?

Observationally answering to those questions is all but an easy task, especially in solar type systems. This is because the bulk of the disk mass resides in the outer midplane, which is cold and dense. As a consequence, all heavy-bearing molecules freeze-out onto the grain mantles, and disappear from the gas phase where they could be observed. So the first difficulty in the study of the outer disk midplane is to find probes of it. Last year, Ceccarelli et al. (2004) detected abundant $\mathrm{H}_{2} \mathrm{D}^{+}$in the proto-planetary disk which surrounds the solar type protostar DM Tau. They claimed that $\mathrm{H}_{2} \mathrm{D}^{+}$probes the cold outer midplane and, in addition, its abundance is a direct measure of the ionization degree. The reason behind this claim is the peculiar chemistry of the $\mathrm{H}_{2} \mathrm{D}^{+}$ion. The basic idea is that in cold and dense gas, where $\mathrm{CO}$ and all heavy-bearing molecules freezeout into the grain mantles, two things happen: first, only $\mathrm{H}_{2}$ and the ions from this molecule, namely $\mathrm{H}_{3}^{+}$and its isotopomers, remain in the gas phase; second, the molecular deuteration is dramatically enhanced, up to having $\mathrm{H}_{2} \mathrm{D}^{+} / \mathrm{H}_{3}^{+}$larger than unity (Caselli et al. 2003). Therefore, Ceccarelli et al. concluded that a) $\mathrm{H}_{2} \mathrm{D}^{+}$line emission probes the gas disk midplane; and b) the $\mathrm{H}_{2} \mathrm{D}^{+}$abundance measures the ionization degree there.

In the present article, we examine the chemistry of the deuterated forms of $\mathrm{H}_{3}^{+}$in proto-planetary disks, with the goal of exploring, on a solid theoretical basis, the exact diagnostic value of the $\mathrm{H}_{2} \mathrm{D}^{+}$observations. Besides, the present study concerns also the other $\mathrm{H}_{3}^{+}$isotopomers, $\mathrm{HD}_{2}^{+}$and $\mathrm{D}_{3}^{+}$. To accomplish the goal, we develop a chemical model of the outer disk midplane, focused in particular on the $\mathrm{H}_{3}^{+}$deuteration chemistry. The physical model that describes the disk computes 
self-consistently the temperature and density profiles for a given disk mass and star luminosity (Dullemond et al. 2001; Dullemond \& Dominik 2004). The chemical model is based on what has been understood from the studies of molecular deuteration in pre-stellar-cores and protostars, both observationally (see e.g. the review by Ceccarelli 2004) and theoretically (e.g. Roberts et al. 2003, 2004; Walmsley et al. 2004; Flower et al. 2004). Particular emphasis is devoted to the role of dust grains, and the effect of dust coagulation/fragmentation on the disk midplane chemical structure.

Aiming to give observable predictions, besides to provide the abundances of the $\mathrm{H}_{3}^{+}$isotopomers across the disk, and the average column density of each species, we compute the intensities of the four ground state lines from the ortho and para forms of $\mathrm{H}_{2} \mathrm{D}^{+}$and $\mathrm{HD}_{2}^{+}$respectively. We carry out a wide parameter study, and explore the dependence of our results on three major parameters: the dust-to-gas ratio, the cosmic rays ionization rate, and the dust grain average sizes. Besides, we also discuss how the results depend on the basic properties of the star-disk system, namely the star luminosity, disk age, mass and radius. Finally, we discuss the case in which $\mathrm{N}_{2}$ disappears from the gas phase simultaneously with $\mathrm{CO}$, which is not what has been observed so far, but what it would be expected based on the laboratory measurements of the $\mathrm{N}_{2}$ and $\mathrm{CO}$ binding energies (e.g. Oberg et al. 2005).

The article is organized as follows. In Sect. 2 we develop our model. In Sect. 3 we report the model predictions of a standard case, and as function of the parameters of the model. In Sect. 4 we discuss the diagnostic values of the observable quantities (line intensities and column densities). Finally, Sect. 5 summarizes the content of the article.

\section{The model}

In this section we describe the model that we developed to calculate the abundance profiles and the average column densities of $\mathrm{H}_{3}^{+}$and its isotopomers across the disk, and the line emission of the two deuterated forms of $\mathrm{H}_{3}^{+}$which have observable ground state rotational transitions, $\mathrm{H}_{2} \mathrm{D}^{+}$and $\mathrm{HD}_{2}^{+}$.

\subsection{Disk structure}

For the model calculations we use a model of passively irradiated hydrostatic flaring circumstellar disks (Dullemond et al. 2001; Dullemond \& Dominik 2004). This model computes the structure (i.e. the density and temperature distribution) in a selfconsistent way. In the center of the disk, a low-mass star is located with a mass of $M_{\star}$ and a luminosity of $L_{\star}$. Around the star we distribute $M_{\text {disk }}$ of material in a disk ranging from $0.1 \mathrm{AU}$ to $R_{\text {disk }}$, with a surface density powerlaw $\Sigma \propto r^{-1}$, implying that the disk mass per unit radius is constant, i.e. both inner and outer disk contain significant amounts of mass. The disk contains dust at a mass fraction of $f_{\mathrm{d} / \mathrm{g}}$ which we assume to be fully mixed with the gas. While in reality, there probably exists a distribution of grain sizes in the disk, we choose a single grains size for the present calculation. This allows us in a simple way to study the effects of grain size. The structure of the disk is then computed by iterating between

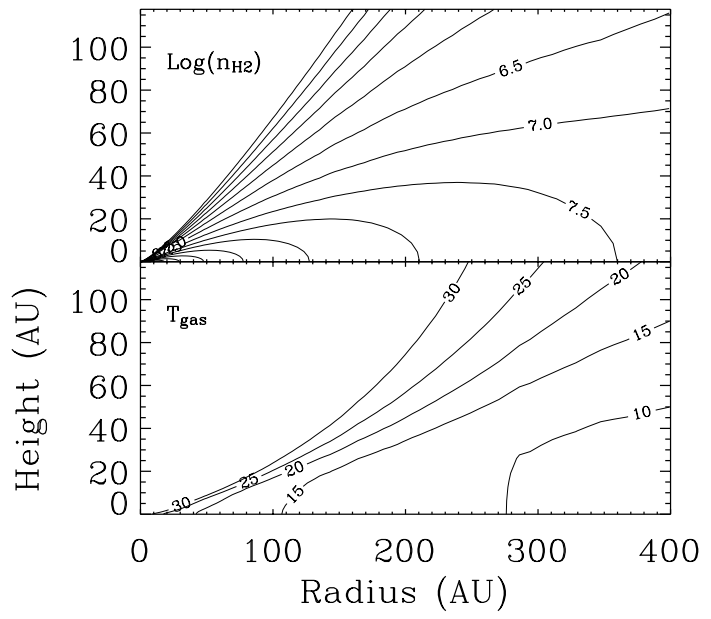

Fig. 1. The density of $\mathrm{H}_{2}$ molecules (top panel), and the local dust temperature in the standard disk model (bottom panel), described in Sect. 3.1.

a $1+1 \mathrm{D}$ continuum radiative transfer which computes the dust temperature in the entire disk, and a hydrostatic equilibrium code which computes the vertical density and pressure distribution under the assumption that the gas and dust temperatures are equal. For the technical details of the modeling procedure we refer to Dullemond et al. (2001). A typical temperature and density profile across the disk (our standard case described in Sect. 3.1) is shown in Fig. 1. The disk is flaring as can be seen from the upwards-curved temperature contours. We anticipate that heavy-elements bearing molecules will freeze-out, and therefore $\mathrm{H}_{3}^{+}$deuterium fractionation will be significant at about $25 \mathrm{~K}$ (approximatively the $\mathrm{CO}$ condensation temperature for the involved densities) and below. These conditions are only fulfilled in the outer disk, approximately outside of $20 \mathrm{AU}$. The low temperature region becomes geometrically thick at large distances from the star. For example, at a distance of $300 \mathrm{AU}$, the $15 \mathrm{~K}$ contour reaches a height of $60 \mathrm{AU}$. Typical densities in this region are $n_{\mathrm{H}_{2}}=10^{7} \mathrm{~cm}^{-3}$ and above, up to $n_{\mathrm{H}_{2}}=10^{8.5} \mathrm{~cm}^{-3}$ in the innermost parts of the outer disk, near $100 \mathrm{AU}$.

\subsection{Chemistry of deuterated $\mathrm{H}_{3}^{+}$}

Complex chemical networks are often necessary to compute the abundances of molecules in interstellar environments. In particular in regions with a rich chemistry, it is difficult to predict which are the important reactions leading to and from a certain molecule (e.g. Semenov et al. 2004). However, in the cold and metal-depleted regions in protostellar cores and circumstellar disks, the chemical network is limited and a much simpler treatment is possible. Also, if the chemical timescales involved are short, a steady state solution of the chemistry is often appropriate. In the following we address the most important processes leading to the formation of deuterated $\mathrm{H}_{3}^{+}$and develop a set of equations for the steady state solution of this network.

In gas under standard molecular cloud conditions, $\mathrm{H}_{3}^{+}$ions are formed with a rate $\zeta$ by the ionization of $\mathrm{H}_{2}$ (and $\mathrm{He}$ ) due to cosmic rays, and destroyed by the reactions with all neutral 
molecules and atoms in the gas, and by recombination reactions with electrons and grains. The reaction $\mathrm{H}_{3}^{+}$with $\mathrm{H}_{2}$ - the most abundant species in molecular gas - returns $\mathrm{H}_{3}^{+}$and therefore has no net effect on the $\mathrm{H}_{3}^{+}$abundance. However, the reaction with $\mathrm{HD}$, the most abundant $\mathrm{H}_{2}$ isotopomer, forms $\mathrm{H}_{2} \mathrm{D}^{+}$:

$\mathrm{H}_{3}^{+}+\mathrm{HD} \rightarrow \mathrm{H}_{2} \mathrm{D}^{+}+\mathrm{H}_{2}$

with a rate coefficient $k_{1}$ (see Table 1 ). The backward reaction is endothermic with an energy barrier of about $220 \mathrm{~K}$. At low temperatures, it is inefficient, making the reaction 1 the effective route to $\mathrm{H}_{2} \mathrm{D}^{+}$formation. When considering the abundances in cold molecular gas, the most important molecules which cause destruction of $\mathrm{H}_{3}^{+}$are: $\mathrm{HD}, \mathrm{CO}$ (the second most abundant molecule after $\mathrm{H}_{2}$ ), and $\mathrm{N}_{2}$ (see Sect. 2.3). Also recombination with grains (see Sect. 2.5) is an important process. Equating $\mathrm{H}_{3}^{+}$formation and destruction rates, and ignoring the destruction by molecules/atoms less abundant than $\mathrm{CO}$ and $\mathrm{N}_{2}$, the equilibrium abundance ratio between the $\mathrm{H}_{3}^{+}$and $\mathrm{H}_{2} \mathrm{D}^{+}$is given by the following equation:

$\frac{\mathrm{H}_{2} \mathrm{D}^{+}}{\mathrm{H}_{3}^{+}}=$

$2 \cdot[\mathrm{D}] k_{1}$

$\overline{k_{\mathrm{rec} 1} x_{\mathrm{e}}+k_{\mathrm{CO}} x_{\mathrm{CO}}+k_{\mathrm{N}_{2}} x_{\mathrm{N}_{2}}+k_{\mathrm{gr}} x_{\mathrm{gr}}+2 \cdot k_{-1}[\mathrm{D}]+2 k_{2}[\mathrm{D}]}$

where the rate coefficients are defined in Table $1, x_{\mathrm{e}}$ is the electronic abundance, and [D] is the elemental abundance of deuterium relative to $\mathrm{H}$ nuclei, equal to $1.5 \times 10^{-5}$ (Lynsky 2003).

As noticed by other authors (e.g. Caselli et al. 2003), the $\mathrm{H}_{2} \mathrm{D}^{+} / \mathrm{H}_{3}^{+}$ratio, given by Eq. (2), can be larger than unity if the gas is cold and very depleted of $\mathrm{CO}$ and $\mathrm{N}_{2}$. In the limit of very cold and completely depleted gas, it reaches $k_{1} / k_{2} \sim 2$ (Walmsley et al. 2004).

Just like $\mathrm{H}_{3}^{+}, \mathrm{H}_{2} \mathrm{D}^{+}$is destroyed by reacting with molecules, atoms, electrons, and grains. In analogy with $\mathrm{H}_{3}^{+}$, the interaction of $\mathrm{H}_{2} \mathrm{D}^{+}$with $\mathrm{HD}$ leads to the formation of $\mathrm{HD}_{2}^{+}$, whereas the reaction with $\mathrm{H}_{2}$ is endothermic and therefore suppressed at low temperatures.

$\mathrm{H}_{2} \mathrm{D}^{+}+\mathrm{HD} \rightarrow \mathrm{HD}_{2}^{+}+\mathrm{H}_{2}$.

As in the case of reaction (1), the reverse reaction of (3) is endothermic, and therefore inhibited at low temperatures. $\mathrm{H}_{2} \mathrm{D}^{+}$is therefore destroyed by reactions with $\mathrm{HD}, \mathrm{CO}, \mathrm{N}_{2}$, electrons, and grains, and forms $\mathrm{HD}_{2}^{+}$. In the same way, the triply deuterated form of $\mathrm{H}_{3}^{+}$is formed by the successive reaction of $\mathrm{HD}_{2}^{+}$with $\mathrm{HD}$ :

$\mathrm{HD}_{2}^{+}+\mathrm{HD} \rightarrow \mathrm{D}_{3}^{+}+\mathrm{H}_{2}$

and destroyed by the reaction with $\mathrm{CO}, \mathrm{N}_{2}$, grains and electrons. Now the reaction $\mathrm{D}_{3}^{+}+\mathrm{HD}$ just exchange the deuterium atoms, and $\mathrm{D}_{3}^{+}$is therefore the dead-end of the chain. As in the case of Eq. (2), the abundance ratios of $\mathrm{HD}_{2}^{+}$and $\mathrm{D}_{3}^{+}$with respect to $\mathrm{H}_{2} \mathrm{D}^{+}$and $\mathrm{HD}_{2}^{+}$respectively can be derived by equating formation and destruction rates:

$\frac{\mathrm{HD}_{2}^{+}}{\mathrm{H}_{2} \mathrm{D}^{+}}=$

$2 \cdot[\mathrm{D}] k_{2}$

$\overline{k_{\mathrm{rec} 2} x_{\mathrm{e}}+k_{\mathrm{CO}} x_{\mathrm{CO}}+k_{\mathrm{N}_{2}} x_{\mathrm{N}_{2}}+k_{\mathrm{gr}} x_{\mathrm{gr}}+2 \cdot k_{-2}[\mathrm{D}]+2 k_{3}[\mathrm{D}]}$
Table 1. Reactions and reaction constants important for the abundance of $\mathrm{H}_{3}^{+}$and its isotopomers. The parameters $\gamma, \alpha$, and $\beta$ determine the rate coefficient at temperature $T$ through $k=\gamma \cdot(T / 300 \mathrm{~K})^{\alpha} \exp \{-\beta / T\}$ and are taken from Roberts et al. (2004) and the UMIST database. A recent study suggests a lower value of the reaction rate of $\mathrm{H}_{3}^{+}$with $\mathrm{HD}$ (Gerlich et al. 2002). However, the study needs confirmation, so we preferred to stick on the old value, which also offers an easier comparison with previous similar models. In the table we only detail the products of those reactions producing $\mathrm{H}_{3}^{+}$and its isotopomers. The destruction reactions involving $\mathrm{CO}, \mathrm{e}^{-}$and grains may have a variety of products. In order to compute the rate coefficients for the reactions with $\mathrm{CO}$ and $\mathrm{e}^{-}$, we added the $\gamma$ parameters of all relevant reactions from the UMIST database. The reaction rates with grains are described in Sect. 2.5.

\begin{tabular}{llllllr}
\hline \hline Reaction & & & Rate & $\gamma$ & $\alpha$ & $\beta$ \\
& & & & $\mathrm{cm}^{3} \mathrm{~s}^{-1}$ & & $\mathrm{~K}$ \\
\hline $\mathrm{H}_{3}^{+}+\mathrm{HD}$ & $\leftrightarrow$ & $\mathrm{H}_{2} \mathrm{D}^{+}+\mathrm{H}_{2}$ & $k_{1}, k_{-1}$ & $1.7(-9)$ & 0.0 & 220 \\
$\mathrm{H}_{3}^{+}+\mathrm{CO}$ & $\rightarrow$ & & $k_{\mathrm{CO}}$ & $6.6(-10)$ & -0.5 & \\
$\mathrm{H}_{3}^{+}+\mathrm{e}^{-}$ & $\rightarrow$ & & $k_{\mathrm{rec} 0}$ & $6.8(-8)$ & -0.5 & \\
$\mathrm{H}_{3}^{+}+\mathrm{g}$ & $\rightarrow$ & & $k_{\mathrm{gr} 1}$ & & & \\
$\mathrm{H}_{2} \mathrm{D}^{+}+\mathrm{HD}$ & $\leftrightarrow$ & $\mathrm{HD}_{2}^{+}+\mathrm{H}_{2}$ & $k_{2}, k_{-2}$ & $8.1(-10)$ & 0.0 & 187 \\
$\mathrm{H}_{2} \mathrm{D}^{+}+\mathrm{CO}$ & $\rightarrow$ & & $k_{\mathrm{CO}}$ & $6.6(-10)$ & -0.5 & \\
$\mathrm{H}_{2} \mathrm{D}^{+}+\mathrm{e}^{-}$ & $\rightarrow$ & & $k_{\mathrm{rec} 1}$ & $6.0(-8)$ & -0.5 & \\
$\mathrm{H}_{2} \mathrm{D}^{+}+\mathrm{g}$ & $\rightarrow$ & & $k_{\mathrm{gr} 2}$ & & & \\
$\mathrm{HD}_{2}^{+}+\mathrm{HD}$ & $\leftrightarrow$ & $\mathrm{D}_{3}^{+}+\mathrm{H}_{2}$ & $k_{3}, k_{-3}$ & $6.4(-10)$ & 0.0 & 234 \\
$\mathrm{HD}_{2}^{+}+\mathrm{CO}$ & $\rightarrow$ & & $k_{\mathrm{CO}}$ & $6.6(-10)$ & -0.5 & \\
$\mathrm{HD}_{2}^{+}+\mathrm{e}^{-}$ & $\rightarrow$ & & $k_{\mathrm{rec} 2}$ & $6.0(-8)$ & -0.5 & \\
$\mathrm{HD}_{2}^{+}+\mathrm{g}$ & $\rightarrow$ & & $k_{\mathrm{gr} 3}$ & & & \\
$\mathrm{D}_{3}^{+}+\mathrm{CO}$ & $\rightarrow$ & & $k_{\mathrm{CO}}$ & $6.6(-10)$ & -0.5 & \\
$\mathrm{D}_{3}^{+}+\mathrm{e}^{-}$ & $\rightarrow$ & & $k_{\mathrm{rec} 3}$ & $2.7(-8)$ & -0.5 & \\
$\mathrm{D}_{3}^{+}+\mathrm{g}$ & $\rightarrow$ & & $k_{\mathrm{gr} 4}$ & & & \\
\hline
\end{tabular}

Notes: $a(-b)$ implies $a \times 10^{-b}$.

$$
\frac{\mathrm{D}_{3}^{+}}{\mathrm{HD}_{2}^{+}}=\frac{2 \cdot[\mathrm{D}] k_{3}}{k_{\mathrm{rec} 3} x_{\mathrm{e}}+k_{\mathrm{CO}} x_{\mathrm{CO}}+k_{\mathrm{N}_{2}} x_{\mathrm{N}_{2}}+k_{\mathrm{gr}} x_{\mathrm{gr}}+2 k_{-3}[\mathrm{D}]}
$$

One can easily see that the $\mathrm{HD}_{2}^{+} / \mathrm{H}_{2} \mathrm{D}^{+}$ratio can reach unity. In the limiting case of very low temperatures, extreme $\mathrm{CO}$ and $\mathrm{N}_{2}$ depletion and low ionization, it reaches a value of $k_{2} / k_{3} \sim 1.3$. On the other hand, the abundance of $\mathrm{D}_{3}^{+}$is only limited by the electron abundance. In extreme conditions - cold and heavily $\mathrm{CO}$ and $\mathrm{N}_{2}$ depleted gas $-\mathrm{D}_{3}^{+}$can be the dominant charge carrier.

Table 1 summarizes the reactions involving $\mathrm{H}_{3}^{+}$and its isotopomers, along with the rates used in this study. The reaction rates have been taken from Roberts et al. (2003, 2004), except for recombination reactions on grains which we discuss in Sect. 2.5.

In practice, the adopted chemical network is a small subset of the extensive chemical networks implemented by Roberts et al. $(2003,2004)$ or Walmsley et al. (2004) or Flower et al. (2004). Our goal here is to study the behavior of the $\mathrm{H}_{3}^{+}$and its isotopomers, which can indeed be described by the few 
equations discussed above. This sets the limit of applicability of the present model to regions where $\mathrm{CO}$ and $\mathrm{N}_{2}$ are the only molecules affecting the $\mathrm{H}_{3}^{+}$chemistry. Furthermore, we did not treat the ortho and para forms independently, but used, when necessary, the results by Walmsley et al. (2004) and Flower et al. (2004).

With this reduced chemical network, we are able to reproduce the results by Roberts et al. (2003, 2004), Walmsley et al. (2004) and Flower et al. (2004) accurately as far as the abundances of the deuterated forms of $\mathrm{H}_{3}^{+}$are concerned.

\section{3. $\mathrm{CO}$ and $\mathrm{N}_{2}$ freeze-out}

The $\mathrm{CO}$ and $\mathrm{N}_{2}$ abundances are obviously critical parameters in the deuteration of $\mathrm{H}_{3}^{+}$. $\mathrm{CO}$ is known to freeze-out onto the grains mantles at large enough densities $\left(\geq 10^{5} \mathrm{~cm}^{-3}\right)$ and low temperatures $(\leq 25 \mathrm{~K})$ (e.g. Caselli et al. 1999; Bergin et al. 2001; Bacmann et al. 2002; Tafalla et al. 2002). Recent laboratory experiments show that the $\mathrm{CO}$ binding energy depends on the matrix where CO is embedded (e.g. Collings et al. 2003; Fraser et al. 2004; Oberg et al. 2005). It varies from $\sim 840 \mathrm{~K}$ in $\mathrm{CO}-\mathrm{CO}$ ices (average of the values measured by Collings et al. 2003 and Oberg et al. 2005), to $885 \mathrm{~K}$ in a CO- $\mathrm{N}_{2}$ ice (Oberg et al. 2005), and $1180 \mathrm{~K}$ in a CO- $\mathrm{H}_{2} \mathrm{O}$ ice (Collings et al. 2003; Fraser et al. 2004). In this study, we will adopt the value $885 \mathrm{~K}$.

There is overwhelming observational evidence, mainly towards pre-stellar cores, that $\mathrm{N}_{2} \mathrm{H}^{+}$remains in the gas phase at larger densities than CO (e.g. Caselli et al. 1999; Bergin et al. 2001; Tafalla et al. 2004; Pagani et al. 2004; Crapsi et al. 2005). Since $\mathrm{N}_{2} \mathrm{H}^{+}$is believed to be formed from $\mathrm{N}_{2}$, these observations suggest that $\mathrm{N}_{2}$, which is the major nitrogen reservoir, freezes-out onto grains at higher densities than $\mathrm{CO}$. The reason for that is not fully understood, because the binding energy of $\mathrm{N}_{2}$, measured in the laboratories, is only slightly lower that the $\mathrm{CO}$ binding energy: $790 \mathrm{~K}$ in a pure $\mathrm{N}_{2}$ ice and $855 \mathrm{~K}$ in a $\mathrm{N}_{2}-\mathrm{CO}$ ice (Oberg et al. 2005). However, the effect of having $\mathrm{N}_{2}$ in the gas phase where $\mathrm{CO}$ molecules disappear greatly influences the abundances of $\mathrm{H}_{3}^{+}$and its isotopomers, because it causes an additional term of $\mathrm{H}_{3}^{+}\left(\mathrm{H}_{2} \mathrm{D}^{+}\right.$and $\left.\mathrm{HD}_{2}^{+}\right)$destruction in Eq. (2) (and Eqs. (5) and (6)). Therefore, we decided to adopt a semi-empirical approach, and to assume that the binding energy of $\mathrm{N}_{2}$ is 0.65 times that of $\mathrm{CO}$, following the theoretical studies of other authors (e.g. Bergin et al. 1995, 1997). However, we will discuss the effect of a larger $\mathrm{N}_{2}$ binding energy, as measured in laboratory, in Sect. 3.4.

We treat the freeze-out of $\mathrm{CO}$ and $\mathrm{N}_{2}$ in a time-dependent way. $\mathrm{CO}$ molecules freeze out onto the grain mantles at a rate:

$k_{\mathrm{dep}}=S\left\langle\pi a_{\mathrm{gr}}^{2} n_{\mathrm{g}}\right\rangle v_{\mathrm{CO}}$

where we adopted a sticking coefficient $S=0.3$ (Burke \& Hollenbach 1983), and a mean grain radius of $a_{\mathrm{gr}}$. The grain number density $n_{\mathrm{g}}$ is given by the (mass) dust-to-gas ratio $f_{\mathrm{d} / \mathrm{g}}$ multiplied by the gas density $n$, and divided by the grain mass (see Eq. (15)). A similar equation can be written for $\mathrm{N}_{2}$.

Frozen $\mathrm{CO}$ and $\mathrm{N}_{2}$ molecules can be released back into the gas phase by thermal evaporation, and we followed the first order desorption kinetics approach to describe it (Hasegawa \& Herbst 1993):

$k_{\mathrm{ev}}=v_{0} \exp \left[-E_{\mathrm{b}} / k T\right]$

where $v_{0}=10^{12} \mathrm{~s}^{-1}$ is the lattice frequency, $E_{\mathrm{b}}$ is the binding energy per molecule of $\mathrm{CO}$ and $\mathrm{N}_{2}$ ice respectively, and $k$ is the Boltzman constant.

Cosmic rays also contribute to the release of $\mathrm{CO}$ and $\mathrm{N}_{2}$ from the ice, with a rate of (Hasegawa \& Herbst 1993):

$k_{\mathrm{cr}}=9.8 \times 10^{-15} \frac{\zeta}{3 \times 10^{17} \mathrm{~s}^{-1}} \mathrm{~s}^{-1}$.

The time dependent number density of gaseous $\mathrm{CO}, n_{\mathrm{CO}}$, is therefore given by the solution of the following equations:

$\frac{\mathrm{d} n_{\mathrm{CO}}}{\mathrm{d} t}=-k_{\mathrm{dep}} n_{\mathrm{CO}}+\left(k_{\mathrm{ev}}+k_{\mathrm{cr}}\right) \cdot n_{\mathrm{CO}}^{\text {ice }}$

$n_{\mathrm{CO}}^{\text {ice }}+n_{\mathrm{CO}}=n_{\mathrm{H}_{2}} \cdot A_{\mathrm{CO}}$

where we adopted the $\mathrm{CO}$ abundance $A_{\mathrm{CO}}$ observed in molecular clouds, $9.5 \times 10^{-5}$ (Frerking et al. 1982). $n_{\mathrm{CO}}^{\text {ice }}$ is the number density of frozen $\mathrm{CO}$ molecules. Similar equations can be written for $\mathrm{N}_{2}$, where the $\mathrm{N}_{2}$ abundance in molecular clouds is assumed to be $4 \times 10^{-5}$ (Bergin et al. 1995, 1997). In our model, the $\mathrm{CO}$ and $\mathrm{N}_{2}$ abundances in the gas phase across the disk depend therefore on the time. They start at time $=0$ equal to their relative molecular cloud abundances. At large times, they are given by the equilibrium between thermal evaporation, cosmic ray desorption and freeze-out onto the mantles (Leger et al. 1986).

Note that we do not treat in any way the $\mathrm{CO}$ and $\mathrm{N}_{2}$ photo dissociation by the UV and/or X-rays photons emitted by the central star. Therefore, our model just describes the regions where those photons are fully shielded, i.e. the warm molecular layer and the cold midplane of sufficiently massive disks. As our model focuses on the $\mathrm{H}_{3}^{+}$and its isotopomers only, it does not have any vocation in treating the $\mathrm{CO}$ and/or $\mathrm{N}_{2}$ chemistry, other than computing their disappearance from the gas phase because of the freezing onto the dust grains. Besides, our model does not consider any possible grain surface chemistry involving $\mathrm{CO}$ and $\mathrm{N}_{2}$, which may transform (part of) condensed $\mathrm{CO}$ (and $\mathrm{N}_{2}$ ) into different, more complex molecules.

\subsection{Charge balance}

Ionization in disks can be due to cosmic rays, $\mathrm{X}$ rays and UV rays. For the current study we restrict ourselves to regions of the disk which are shielded from UV radiation and X rays, i.e. to the disk midplane. In this case, cosmic rays are the dominating source of ionization (Semenov et al. 2004).

In regions where the heavy-elements bearing molecules are depleted, the positive charge is carried by the H-bearing species, namely $\mathrm{H}_{3}^{+}$, its isotopomers, and $\mathrm{H}^{+}$. The negative charge, on the other hand, is mostly carried by free electrons. The fraction of electrons attached to grains remains small, in particular if the smallest grains have been removed by coagulation (Walmsley et al. 2004). We will therefore ignore negatively charged grains when computing the abundances of $\mathrm{H}^{+}$, $\mathrm{H}_{3}^{+}$(with isotopomers), and $\mathrm{e}^{-}$. 
Similar to $\mathrm{H}_{3}^{+}$(see Sect. 2.2) $\mathrm{H}^{+}$is formed by the interaction of cosmic rays with $\mathrm{H}_{2}$, with a rate $0.08 \zeta$. Recombination of $\mathrm{H}^{+}$occurs mainly on grains because the direct recombination with electrons is too slow, and the proton exchange with HD. The abundance of $\mathrm{H}^{+}$is therefore independent of the electron density. Equating the formation and destruction rates, we have:

$x_{\mathrm{H}^{+}}=\frac{0.08 \zeta}{2 k_{\mathrm{HD}}[\mathrm{D}]+k_{\mathrm{gr}} x_{\mathrm{gr}}}$

where $\zeta$ is the cosmic ray ionization rate. The recombination rate on grains $k_{\mathrm{gr}}$ is discussed in Sect. 2.5.

The electron abundance may be derived by equating the cosmic ray ionization rates with the processes removing electrons from the gas. As source term of free electrons we consider the reactions $\mathrm{H}_{2}+\mathrm{cr} \rightarrow \mathrm{H}_{3}^{+}+\mathrm{e}^{-}$and $\mathrm{H}_{2}+\mathrm{cr} \rightarrow \mathrm{H}^{+}+\mathrm{e}^{-}$. As electron sink term we use the recombination of $\mathrm{H}_{3}^{+}$and its isotopes with electrons, with a dissociative recombination rate $\beta$, measured by McCall et al. (2003) to be $4 \times 10^{-7}$ at $10 \mathrm{~K}$. In addition, we assume that every recombination of $\mathrm{H}_{3}^{+}$(and its isotopomers) and $\mathrm{H}^{+}$on grains remove an electron from the gas. This is a reasonable assumption because positively charged grains quickly recombine with free electrons, consistent with the very low abundance of positively charged grains found by (Walmsley et al. 2004). This leads to the following balance equation for the electron density:

$\zeta(1.08) n_{\mathrm{H}_{2}}=\left(\beta x_{\{\mathrm{H}, \mathrm{D}\}_{3}^{+}}+k_{\mathrm{gr}} x_{\mathrm{H}^{+}}+\frac{k_{\mathrm{gr}}}{\sqrt{m / m_{\mathrm{H}}}} x_{\{\mathrm{H}, \mathrm{D}\}_{3}^{+}}\right) n_{\mathrm{H}_{2}} n_{\mathrm{e}^{-}}$

where $x_{\langle\mathrm{H}, \mathrm{D}\}_{3}^{+}}$is the total abundance of $\mathrm{H}_{3}^{+}$and its isotopomers, and $m$ is the average mass of these molecules which we set equal to $4.5 m_{\mathrm{H}}$. Together with charge conservation $x_{\mathrm{e}^{-}}=x_{\mathrm{H}^{+}}+$ $x_{\mathrm{H}_{3}^{+}}+x_{\mathrm{H}_{2} \mathrm{D}^{+}}+x_{\mathrm{D}_{2} \mathrm{H}^{+}}+x_{\mathrm{D}_{3}^{+}}$, a 2nd degree equation for the electron density results which can be easily solved.

We can consider two limiting cases. If the electron abundance is set by the recombination of electrons with $\mathrm{H}_{3}^{+}$and its isotopes, we can expect the canonical dependence $x_{\mathrm{e}^{-}} \propto$ $\sqrt{\zeta / n_{\mathrm{H}_{2}}}$. If on the other hand the electron abundance is set by recombination of $\mathrm{H}^{+}$on grains, the electron abundance will be $x_{\mathrm{e}^{-}} \propto \zeta / n_{\mathrm{gr}}$ where $n_{\mathrm{gr}}$ is the number density of grains. We will use this difference below to identify the main recombination process in the model calculations.

\subsection{Recombination on grains}

At low electron densities, collisions of ions with grains become an important contributer to the recombination rates. This is true in particular for protons, and, depending on the rate coefficients for dissociative recombination, also for molecular ions. At high densities, the charge distribution is dominated by neutral and singly negatively charged grains. Draine \& Sutin (1987) argue that the sticking coefficients for positive ions on both neutral and negatively charged grains should be 1 . The different recombination rates then only depend on the Coulomb focusing factor in the collision cross section. The Coulomb focusing factor $\tilde{J}$ is given by Draine \& Sutin (1987) as a function of the grain charge, ion charge and the gas temperature. For a full treatment, the relative abundances of neutral and negatively charged

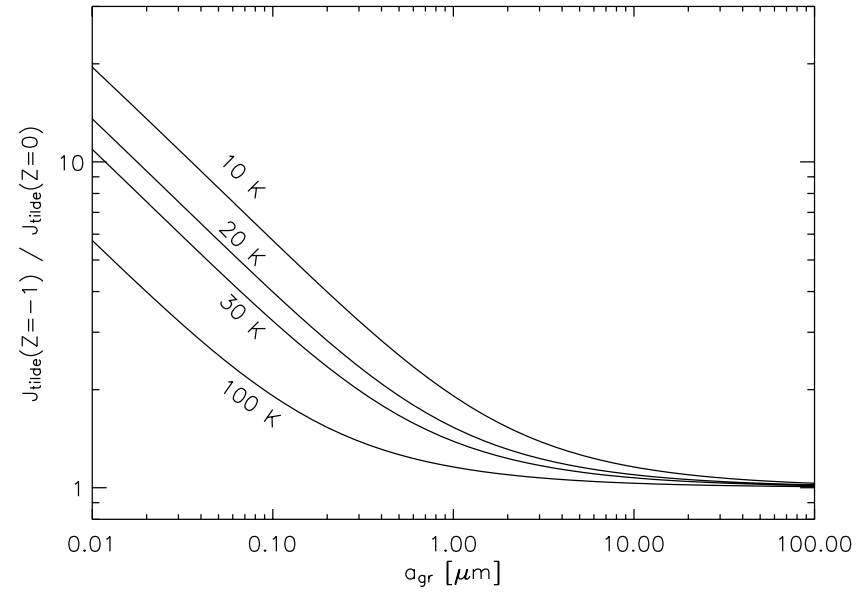

Fig. 2. Ratio $\tilde{J}\left(Z_{\mathrm{gr}}=-1\right) / \tilde{J}\left(Z_{\mathrm{gr}}=0\right)$ as function of the grain radius, for different temperatures (see text).

grains would have to be calculated, as e.g. done by (Walmsley et al. 2004). However, in the case of really dense environments, in which the abundance of small grains is small due to the effects of dust coagulation, a simplified treatment is possible. Figure 2 shows the ratio $\tilde{J}\left(Z_{\mathrm{gr}}=-1\right) / \tilde{J}\left(Z_{\mathrm{gr}}=0\right)$. While for very small grain sizes and extremely low temperatures this ratio can be very large, in the region of parameter space studied in this paper the factor is reasonably small. For $0.1 \mu \mathrm{m}$, the ratio is already down to 5.7. For larger grains and somewhat higher temperature, the ratio gets closer to one. For simplicity we therefore choose the following approach. We assume that a fraction $f_{-}$of the grains is negatively charged, and that $1-f_{-}$ of the grains are neutral. The recombination rate coefficient $k_{\mathrm{gr}}$ is then given by (Draine \& Sutin 1987):

$k_{\mathrm{gr}}=\left(\frac{8 k T}{\pi m_{\mathrm{H}}}\right)^{1 / 2} \pi a_{\mathrm{gr}}^{2}\left(f_{-} \tilde{J}(Z=-1)+\left(1-f_{-}\right) \tilde{J}(Z=0)\right)$.

Choosing $f_{-}=0.3$, we expect an error in the recombination rates from this approach no larger than a factor of 3 .

Finally, because of the uncertainties of the grain size distribution in the midplane of a disk, we use only a single grain size $a_{\mathrm{gr}}$ for each model. Assuming a density of the grain material of $2.5 \mathrm{gr} \mathrm{cm}^{-3}$, the dust-to-grain ratio in number density is given by:

$x_{\mathrm{gr}}=3.2 \times 10^{-12}\left(\frac{f_{\mathrm{d} / \mathrm{g}}}{0.01}\right)\left(\frac{a_{\mathrm{gr}}}{0.1 \mu \mathrm{m}}\right)^{-3}$.

\section{6. $\mathrm{H}_{2} \mathrm{D}^{+}$and $\mathrm{D}_{2} \mathrm{H}^{+}$line intensities}

$\mathrm{H}_{2} \mathrm{D}^{+}$and $\mathrm{D}_{2} \mathrm{H}^{+}$both appear in the para and ortho forms. Gerlich et al. Roueff (2002) have discussed in detail the issue, and found that at low temperatures the ortho to para ratio of $\mathrm{H}_{2} \mathrm{D}^{+}$is close to unity. Subsequently, Walmsley et al. (2004) have shown that the $\mathrm{H}_{2} \mathrm{D}^{+}$ortho to para ratio indeed varies weakly with the density in completely depleted regions, and it is around 0.3 at densities $\geq 10^{6} \mathrm{~cm}^{-3}$. Since the present study focuses on the outer disk midplane where the densities are larger than $10^{6} \mathrm{~cm}^{-3}$, we do not calculate the $\mathrm{H}_{2} \mathrm{D}^{+}$ortho-to-para ratio from a chemical network, but simply assume it to be 0.3 . 
Finally, Flower et al. (2004) find that the $\mathrm{HD}_{2}^{+}$ortho-to-para ratio is equal to 10 for large densities and low temperatures.

The ortho and para forms of both $\mathrm{H}_{2} \mathrm{D}^{+}$and $\mathrm{HD}_{2}^{+}$have ground transitions in the submillimeter to Far-IR (Tera-Hertz) range (Hirao \& Amano 2003): 372.4 and $1370.1 \mathrm{GHz}$ for the $\mathrm{o}-\mathrm{H}_{2} \mathrm{D}^{+}$and $\mathrm{p}-\mathrm{H}_{2} \mathrm{D}^{+}$respectively, and 1476.6 and $691.7 \mathrm{GHz}$ for the $\mathrm{o}-\mathrm{HD}_{2}^{+}$and $\mathrm{p}-\mathrm{HD}_{2}^{+}$, respectively. The $\mathrm{o}-\mathrm{H}_{2} \mathrm{D}^{+}$and $\mathrm{p}-\mathrm{HD}_{2}^{+}$transitions are observable with ground based telescopes (CSO and JCMT today, APEX and ALMA in the near future), whereas the other two transitions can only be observed with airborne and satellite telescopes, in particular SOFIA and the upcoming HERSCHEL mission. In this study, we report the line intensity of the four transitions, computed with a non-LTE code which treats self-consistently the line optical depth (Ceccarelli et al. 2003) ${ }^{1}$, assuming that the disk is seen face-on. Note that, for the disks studied in this article, the line optical depth in the face-on configuration never exceeds unity though, so that the lines are in practice always optically thin. The collisional coefficients are not well known, and we assumed that the critical density of all the $\mathrm{H}_{2} \mathrm{D}^{+}$and $\mathrm{HD}_{2}^{+}$ground transitions are $10^{6} \mathrm{~cm}^{-3}$ derived using the $\mathrm{H}_{2} \mathrm{D}^{+}$scaled collisional coefficients by Black et al. (1990). Since the regions where the lines originate have relatively large densities $\left(\geq 10^{7} \mathrm{~cm}^{-3}\right)$, the levels are mostly LTE populated, and the uncertainty on the collisional coefficients is, therefore, not of great importance here. The line fluxes are given in erg $\mathrm{s}^{-1} \mathrm{~cm}^{-2}$ assuming a source distance of $140 \mathrm{pc}$. For an easy comparison with the presently available observations, we also give the $\mathrm{o}-\mathrm{H}_{2} \mathrm{D}^{+}$(at $372 \mathrm{GHz}$ ) and p- $\mathrm{HD}_{2}^{+}$(at $691 \mathrm{GHz}$ ) line fluxes in main beam temperature, assuming that the observations are carried out at the CSO and JCMT telescopes respectively.

\section{Results}

In this section we discuss the results of the model calculations, namely the abundances of the $\mathrm{H}_{2} \mathrm{D}^{+}, \mathrm{HD}_{2}^{+}$and $\mathrm{D}_{3}^{+}$across the disk. One major goal of this article is to give predictions for observations of the two deuterated forms of $\mathrm{H}_{3}^{+}$that have ground rotational transitions in the submillimeter wavelength range: $\mathrm{H}_{2} \mathrm{D}^{+}$and $\mathrm{D}_{2} \mathrm{H}^{+}$.

We first explore in detail our standard case, which is a disk with canonical values for parameters like dust-to-gas ratio, cosmic ray flux and grain sizes, properties not easily derived from observations. In Sect. 3.2 we study the dependence on these parameters. In Sect. 3.3 we address system properties that are generally known from other observations: the total dust mass, the disk size, and the stellar luminosity. Finally, in Sect. 3.4 we discuss the influence of one important model assumption, the binding energy of $\mathrm{N}_{2}$ and $\mathrm{CO}$ ice.

\subsection{The standard model}

Our standard model is a disk with a dust disk mass equal to $2 \times 10^{-4} M_{\odot}$ surrounding a $0.5 L_{\odot}$ star whose $T_{\text {eff }}=3630 \mathrm{~K}$ (see Sect. 2.1). The dust-to-gas ratio is $1 / 100$ in mass, and we use the canonical cosmic ray ionization rate $\zeta=3 \times 10^{-17} \mathrm{~s}^{-1}$.

\footnotetext{
1 The code has been adapted to the disk geometry.
}

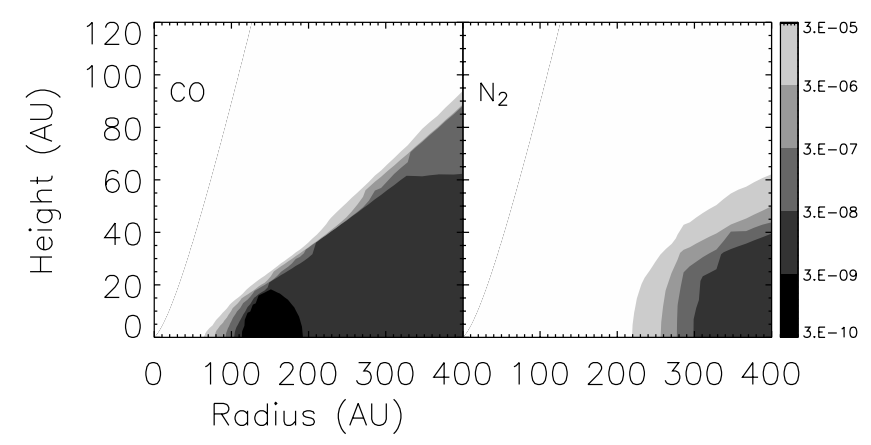

Fig. 3. Abundance profiles of $\mathrm{CO}$ (right panel) and $\mathrm{N}_{2}$ (left panel) across the disk, for our standard case. The different colors show the abundance as given by the scale bar.

We assume that the disk is $10^{6} \mathrm{yr}$ old, and extends up to $400 \mathrm{AU}$. Figure 1 shows the density and temperature profiles across the disk of our standard case.

Figure 3 shows the abundances of $\mathrm{CO}$ and $\mathrm{N}_{2}$ respectively, across the disk. $\mathrm{CO}$ molecules disappear from the gas phase where the dust temperature is around $25 \mathrm{~K}$, at a radius larger than about $100 \mathrm{AU}$ and at heights lower than about $1 / 4$ of the radius. Since the density is large in those cold regions, the drop in the abundance is very sharp, more than a factor 100 . The largest CO depletion occurs between about 100 and $200 \mathrm{AU}$, where it is larger than $10^{4}$ times. At larger radii it is a factor 10 lower. $\mathrm{N}_{2}$, however, remains in the gas phase in a much larger region, and, therefore, dominates the destruction rates of $\mathrm{H}_{3}^{+}$(and its isotopomers). It is only in the very outer regions, at a radius larger than about $300 \mathrm{AU}$ that $\mathrm{N}_{2}$ freezes-out onto the grains and almost totally disappears from the gas phase. The intermediate region, where $\mathrm{CO}$ is depleted but $\mathrm{N}_{2}$ is not, is where the deuteration of $\mathrm{H}_{3}^{+}$takes place in a "moderate" form, namely where the $\mathrm{H}_{2} \mathrm{D}^{+} / \mathrm{H}_{3}^{+}$ratio is enhanced with respect to the elemental $\mathrm{D} / \mathrm{H}$ abundance, but $\mathrm{D}_{3}^{+}$is not the dominant positive charge carrier.

Figure 4 shows the $\mathrm{H}^{+}, \mathrm{H}_{3}^{+}$isotopomers, and electron abundance profiles of the standard disk respectively. We limit the plot of the chemical composition to regions where the $\mathrm{CO}$ depletion is larger than a factor of 3 . The choice of 3 is a somewhat arbitrary. It represents when the $\mathrm{CO}$ abundance is similar to that of $\mathrm{N}_{2}$, or, in other words, when the role of these two molecules - believed to be the most abundant gaseous molecules at low temperatures - becomes of similar importance in the $\mathrm{H}_{3}^{+}$reactions, the $\mathrm{H}_{3}^{+}$deuteration becomes to be significant, and our simplified model correct. The electron abundance, is between 3 and $30 \times 10^{-10}$ in the outermost region, at radius $\geq 50 \mathrm{AU}$, increasing going towards the upper layers of the disk. The results are in reasonable agreement with the ionization structure computed by Semenov et al. (2004). In most of the disk, the positive charge is carried by $\mathrm{H}_{3}^{+}$, which becomes less important where also the $\mathrm{N}_{2}$ disappears from the gas phase, at radius $\geq 300 \mathrm{AU} . \mathrm{H}_{2} \mathrm{D}^{+}$is the most abundant $\mathrm{H}_{3}^{+}$isotope, except in the radius $\geq 300 \mathrm{AU}$ region, where $\mathrm{D}_{3}^{+}$takes over, and become the most abundant positive ion. $\mathrm{H}^{+}$never plays a major role as charge carrier across the entire disk, even though its abundance is comparable to that of $\mathrm{H}_{2} \mathrm{D}^{+}$in outer upper levels of the disk. 


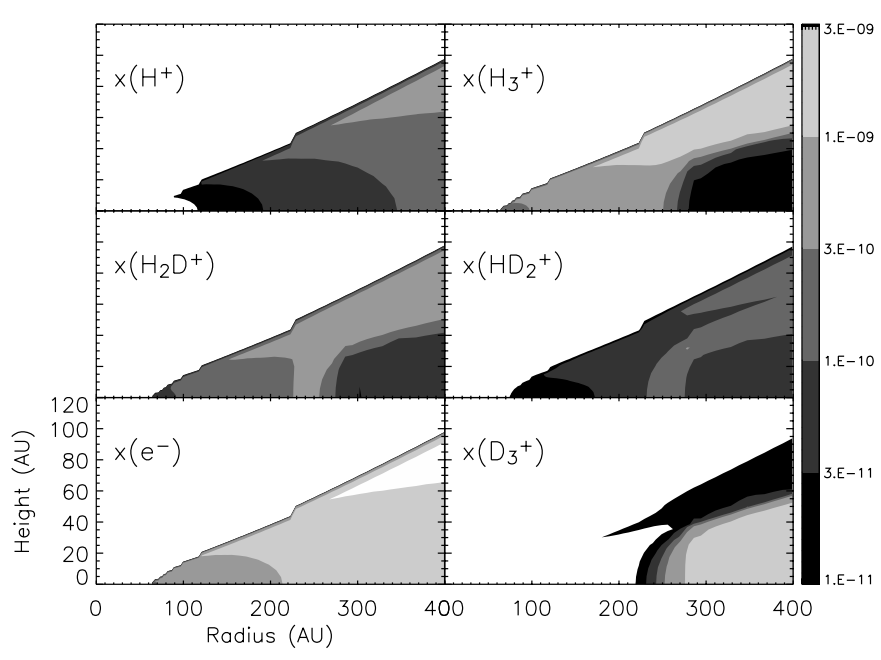

Fig. 4. Chemical structure of the standard disk: fractional abundances with respect to $\mathrm{H}_{2}$ as function of the disk radius and height, in AU. Top left panel: $\mathrm{H}_{3}^{+}$. Top right panel: $\mathrm{H}^{+}$. Center left panel: $\mathrm{H}_{2} \mathrm{D}^{+}$. Center right panel: $\mathrm{HD}_{2}^{+}$. Bottom left panel: electrons. Bottom right panel: $\mathrm{D}_{3}^{+}$. The color levels show the abundance as given by the scale bar.

Table 2. Column densities averages across the face-on disk of the $\mathrm{H}_{3}^{+}$isotopes and electrons in the standard disk model.

\begin{tabular}{lc}
\hline \hline Species & Column density $\left(\mathrm{cm}^{-2}\right)$ \\
\hline $\mathrm{H}_{2} \mathrm{D}^{+}$ & $1.6 \times 10^{13}$ \\
$\mathrm{HD}_{2}^{+}$ & $5.1 \times 10^{12}$ \\
$\mathrm{D}_{3}^{+}$ & $3.3 \times 10^{13}$ \\
$\mathrm{e}^{-}$ & $1.0 \times 10^{14}$ \\
\hline
\end{tabular}

The average column densities of a face-on disk of the $\mathrm{H}_{3}^{+}$isotopes and the electrons in the region where the $\mathrm{CO}$ depletion is larger than 3 are reported in Table 2 . The $\mathrm{D}_{3}^{+}$column density is the largest, and it is about $30 \%$ larger that of $\mathrm{H}_{2} \mathrm{D}^{+}$. The two isotopes together account for almost half of the overall positive charge in the disk midplane. The $\mathrm{HD}_{2}^{+}$column density is twice smaller than the $\mathrm{H}_{2} \mathrm{D}^{+}$column density. These values are dominated by the outer region, where both $\mathrm{CO}$ and $\mathrm{N}_{2}$ are frozen-out onto the grains, and hence, $\mathrm{D}_{3}^{+}$becomes the dominant positive charge carrier.

The line intensities of the four ortho and para $\mathrm{H}_{2} \mathrm{D}^{+}$and the $\mathrm{HD}_{2}^{+}$ground transitions are reported in Table 3 .

\subsection{Ionization rate, dust-to-gas ratio and grain sizes as important factors for the abundances}

Important parameters of the model are the cosmic ray ionization rate, the dust-to-gas ratio, and the grain sizes, all of which are relatively ill known. One goal of this theoretical work is to provide predictions of observable quantities which can help to constrain these parameters, which all play a key role in the evolution of the proto-planetary disks and the eventual planet formation (see Introduction).

In order to investigate the influence of the cosmic ray flux and of the dust-to-gas ratio, we have computed a grid of models
Table 3. Line fluxes of the ground transitions of the ortho and para form of $\mathrm{H}_{2} \mathrm{D}^{+}$and the $\mathrm{HD}_{2}^{+}$respectively, for the standard case. The velocity-integrated line intensities, expressed in main beam temperatures, $T_{\mathrm{mb}} \Delta v$ are computed assuming observations at CSO and JCMT of the $\mathrm{o}-\mathrm{H}_{2} \mathrm{D}^{+}$and $\mathrm{p}-\mathrm{HD}_{2}^{+}$transitions respectively.

\begin{tabular}{l|cccc}
\hline \hline & $\mathrm{o}-\mathrm{H}_{2} \mathrm{D}^{+}$ & $\mathrm{p}-\mathrm{H}_{2} \mathrm{D}^{+}$ & $\mathrm{o}-\mathrm{HD}_{2}^{+}$ & $\mathrm{p}-\mathrm{HD}_{2}^{+}$ \\
\hline Transition & $1_{1,0}-1_{1,1}$ & $1_{0,1}-0_{0,0}$ & $1_{1,1}-0_{0,0}$ & $1_{1,0}-1_{0,1}$ \\
$v(\mathrm{GHz})$ & 372.4 & 1370.1 & 1476.6 & 691.7 \\
Flux erg $/ \mathrm{s} / \mathrm{cm}^{2}$ & $8.2 \mathrm{e}-18$ & $1.0 \mathrm{e}-16$ & $5.7 \mathrm{e}-17$ & $2.6 \mathrm{e}-18$ \\
$T_{\mathrm{mb}} \Delta v\left(\mathrm{mK} \mathrm{km} \mathrm{s}^{-1}\right)$ & $18.8^{a}$ & - & - & $4.8^{a}$ \\
\hline
\end{tabular}

${ }^{a}$ Note: the main beam efficiency is assumed to be 0.6 at CSO and 0.3 at JCMT.

where we vary these parameters within a factor 100 of the standard value. Since the structure of the disk is fully determined by the dust opacity, changing the dust-to-gas ratio simply implies scaling the gas density while all other factors like gas temperature and grain number density remain constant. Figure 5 shows the column densities as a function of the cosmic ray ionization rate normalized to $3 \times 10^{-17}$, and of the dust-to-gas ratio. It is immediately obvious that the electron column density varies as a simple powerlaw $\propto \sqrt{\zeta / f_{\mathrm{d} / \mathrm{g}}} \propto \sqrt{\zeta n_{\mathrm{H}_{2}}}$. This corresponds to an electron abundance dependence $x_{\mathrm{e}^{-}} \propto \sqrt{\zeta / n_{\mathrm{H}_{2}}}$, indicating that cosmic ray ionization and dissociative recombination of electrons with the charge carrying molecules $\left(\mathrm{H}_{3}^{+}\right.$and its isotopes in this case) are determining the electron abundance. This is consistent with the isotopes of $\mathrm{H}_{3}^{+}$being the main charge carriers. Because throughout the plot, the dust grain density $n_{\mathrm{gr}}$ is constant (changing $f_{\mathrm{d} / \mathrm{g}}$ only changes the gas density), recombination on grains would lead to a dependence $x_{\mathrm{e}^{-}} \propto \zeta / n_{\mathrm{H}_{2}}$.

The shape of the contours for the column densities of $\mathrm{H}_{2} \mathrm{D}^{+}$ and $\mathrm{D}_{2} \mathrm{H}^{+}$are similar to those of $\mathrm{e}^{-}$, again indicating that these molecules are the dominant charge carriers. $\mathrm{D}_{3}^{+}$shows a similar behavior for very low dust-to-gas ratios (i.e. high gas densities) and normal or low cosmic ray ionization rates. However, for very low gas densities (high dust-to-gas ratios) and for very high ionization rates, the contours become vertical, indicating that the column density becomes independent of the ionization rate. A similar but smaller effect can also be observed for the column densities of $\mathrm{H}_{2} \mathrm{D}^{+}$and $\mathrm{HD}_{2}^{+}$at high dust-to-gas ratios. This is due to the fact that at low gas densities (corresponding also to low electron densities), the recombination of these molecules on grains starts to become important and leads to a faster decrease in the abundances.

Figures 6 to 7 are similar to Fig. 5, but using a disk model with different grains sizes. We have used grain sizes of $0.01 \mu \mathrm{m}$, and $1.0 \mu \mathrm{m}$, to study the cases of very little dust growth compared to the interstellar medium, and significant dust growth. Since the total dust mass in the model is constant, reducing the grain size corresponds to an increase in the number density of grains, and also in the total grain surface per $\mathrm{cm}^{3}$. This does favor recombination on grains as a destruction route of $\mathrm{H}_{3}^{+}$and its isotopes. This is indeed visible, in particular at high dust-to-gas ratios, the column densities of all three molecules are strongly decreased, and almost independent of the ionization rate. 


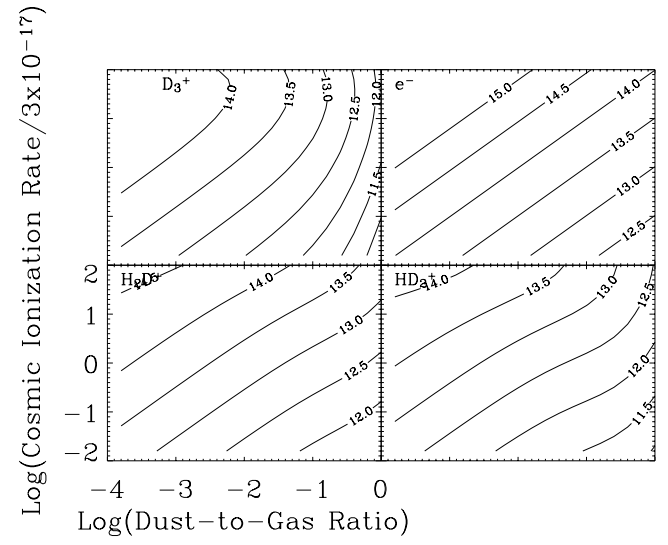

Fig. 5. Logarithm of the column density averaged over the disk surface of $\mathrm{D}_{3}^{+}$(upper left panel), $\mathrm{H}_{2} \mathrm{D}^{+}$(lower left panel), $\mathrm{HD}_{2}^{+}$(lower right panel) and electrons (upper right panel). The plots have been obtained for our standard case, namely with a grain radius of $0.1 \mu \mathrm{m}$.

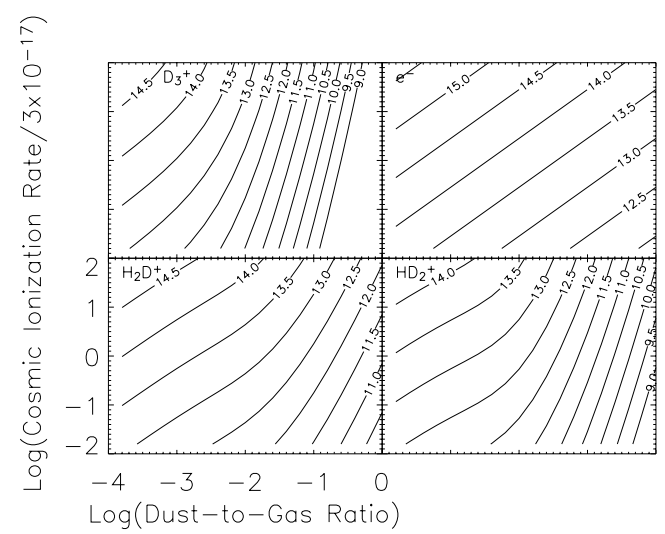

Fig. 6. Same as Fig. 5 but for a grain radius of $0.01 \mu \mathrm{m}$.

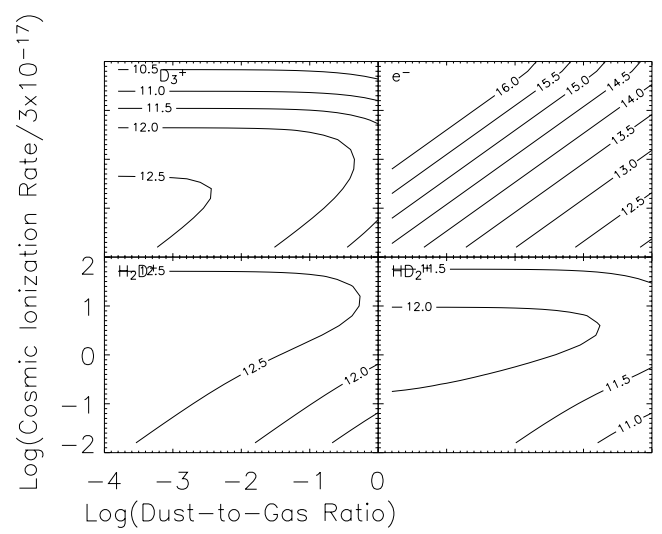

Fig. 7. Same as Fig. 5 but for a grain radius of $1.0 \mu \mathrm{m}$.

A different effect can be observed in the calculation for large grains. In this case, the column density of electrons greatly exceeds that of the $\mathrm{H}_{3}^{+}$isotopes, in particular in the upper left part of the diagram, at high ionization rates and high gas densities. The electron column density contours are closer together, indicating a linear dependence on ionization rate and gas density. As discussed in Sect. 2.4, this is an indication that $\mathrm{H}^{+}$is now the dominating positive charge carrier and that recombination of $\mathrm{H}^{+}$on grains has taken over as main electron

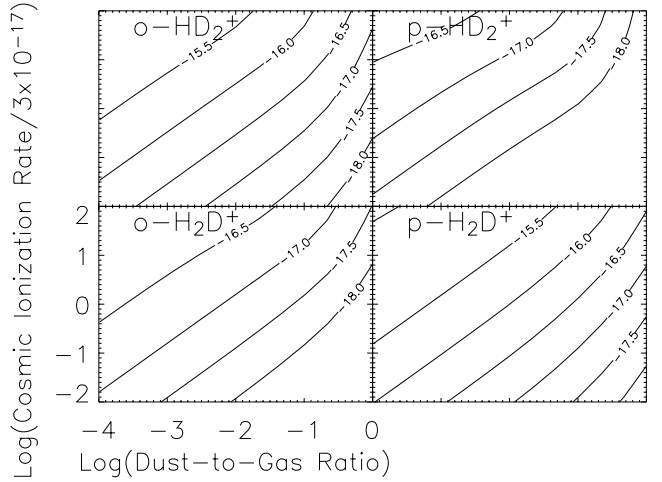

Fig. 8. Logarithm of the line fluxes as function of the dust-to-gas ratio and the cosmic ray ionization rate. The fluxes are in $\mathrm{erg} / \mathrm{s} / \mathrm{cm}^{2}$ and computed for a source at $140 \mathrm{pc}$ of distance. The four panels show the four observable transitions: $\mathrm{o}-\mathrm{H}_{2} \mathrm{D}^{+}(372 \mathrm{GHz}), \mathrm{p}-\mathrm{H}_{2} \mathrm{D}^{+}(1370 \mathrm{GHz})$, $\mathrm{o}-\mathrm{HD}_{2}^{+}(1476 \mathrm{GHz})$ and $\mathrm{p}-\mathrm{HD}_{2}^{+}(691 \mathrm{GHz})$.

destruction mechanism. The reason for the significant decrease in deuterated $\mathrm{H}_{3}^{+}$abundance compared to cases with smaller grains is caused by smaller $\mathrm{CO} / \mathrm{N}_{2}$ depletions. The larger grains provide less grain surface for these molecules to freeze out and shift the abundance ratios away from the deuterated species towards $\mathrm{H}_{3}^{+}$. The column densities of $\mathrm{H}_{2} \mathrm{D}^{+}, \mathrm{HD}_{2}^{+}$and $\mathrm{D}_{3}^{+}$all have a maximum at normal cosmic ray ionization rates. If the cosmic ray flux is further increased, the column densities degrease again, because the $\mathrm{CO} / \mathrm{N}_{2}$ depletion becomes less efficient. Also for smaller grains, the same effect is expected, but at much higher fluxes.

Figure 8 shows the dependence of the $\mathrm{H}_{2} \mathrm{D}^{+}$and $\mathrm{HD}_{2}^{+}$line fluxes on the cosmic ray ionization rate and the dust-to-gas ratio. For an easy comparison with observations obtainable at CSO and JCMT we also show plots with the velocityintegrated mean beam temperatures of the o- $\mathrm{HD}_{2}^{+}$at $372 \mathrm{GHz}$ and p- $\mathrm{HD}_{2}^{+}$at $691 \mathrm{GHz}$ lines respectively (Fig. 9). The curves of the $\mathrm{o}-\mathrm{H}_{2} \mathrm{D}^{+}$and $\mathrm{p}-\mathrm{HD}_{2}^{+}$line intensities run practically parallel, so that the simultaneous observation of the two lines would not depend on the parameters of the model, but the ortho-topara ratio of each species. They can therefore be "safely" used to derive the relative ortho-to-para ratio of $\mathrm{H}_{2} \mathrm{D}^{+}$with respect to $\mathrm{HD}_{2}^{+}$.

\subsection{The basic stellar and disk properties}

The basic stellar and disk parameters such as stellar luminosity, dust mass in the disk and disk size can normally derived independently. We therefore do not enter into a full parameter study of these quantities. Instead, we limit ourselves to describe the main trends by looking at single-parameter changes from the standard model. Table 4 summarizes the results of these calculations. The stellar and disk parameters mainly change the local densities in the disk, or the temperature. Temperature increase influences the excitation of the lines, and may reduce depletion. Density changes modify the degree of ionization and again the depletion efficiency. The results, summarized in Table 4, show that changes of a factor of two in any of the parameters have only limited effects on the line fluxes, in all cases less than a 


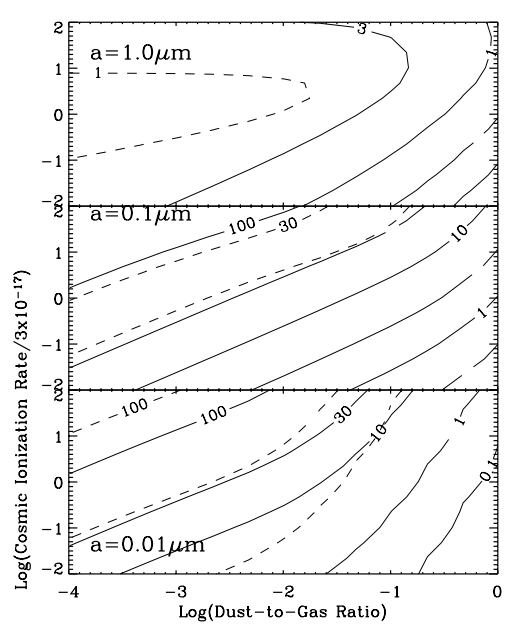

Fig. 9. Velocity-integrated line intensities, expressed as mean beam temperature, of the o- $\mathrm{HD}_{2}^{+} 1_{1,0}-1_{1,1}$ (solid lines) and $\mathrm{p}-\mathrm{HD}_{2}^{+} 1_{1,0}-1_{0,1}$ (dashed lines) transitions for CSO and JCMT observations respectively. The contour plots are in $\mathrm{mK} \mathrm{km} \mathrm{s}{ }^{-1}$. Upper, center and lower panels refer to different grain radii: $0.01,0.1$ and $1.0 \mu \mathrm{m}$ respectively.

Table 4. Changes of the line fluxes emitted by the disk for different values of the stellar and disk parameters. The numbers are the fluxes of the four ground ortho and para $\mathrm{H}_{2} \mathrm{D}^{+}$and $\mathrm{HD}_{2}^{+}$lines relative to the standard case.

\begin{tabular}{l|rccc}
\hline \hline Parameter & o- $\mathrm{H}_{2} \mathrm{D}^{+}$ & $\mathrm{p}-\mathrm{H}_{2} \mathrm{D}^{+}$ & $\mathrm{o}-\mathrm{HD}_{2}^{+}$ & $\mathrm{p}-\mathrm{HD}_{2}^{+}$ \\
\hline$L_{\star} \times 2$ & 1.21 & 1.46 & 1.23 & 0.86 \\
$L_{\star} / 2$ & 0.73 & 0.73 & 0.73 & 0.77 \\
$M_{\text {dust }} \times 2$ & 1.44 & 1.45 & 1.46 & 1.46 \\
$M_{\text {dust }} / 2$ & 0.75 & 0.82 & 0.73 & 0.60 \\
$R_{\text {disk }} \times 2$ & 1.64 & 1.43 & 1.38 & 1.65 \\
$R_{\text {disk }} / 2$ & 0.51 & 0.73 & 0.65 & 0.40 \\
\hline
\end{tabular}

factor of two. The largest changes occur when the disk radius is changed, mostly because of the larger/smaller integration emitting region.

\subsection{High $\mathrm{N}_{2}$ binding energy}

As mentioned in Sect. 2.3, there is a discrepancy between the measured binding energy of $\mathrm{N}_{2}$ onto the grains - which is similar to the $\mathrm{CO}$ binding energy - and the observations in prestellar cores - which show that $\mathrm{N}_{2}$ molecules condense onto the grains at a larger density, and therefore, suggest a $\mathrm{N}_{2}$ binding energy lower than the $\mathrm{CO}$ one. In our standard model, discussed in the previous paragraphs, we adopted that the $\mathrm{N}_{2}$ binding energy is 0.65 times that of $\mathrm{CO}$, in agreement with the modeling of the pre-stellar cores observations (e.g. Bergin et al. 1995, 1997). It is also worth noticing that observations of $\mathrm{N}_{2} \mathrm{H}^{+}$in a proto-planetary disk support indeed the lower binding energy for $\mathrm{N}_{2}$ (Qi et al. 2003). However, for the sake of completeness, here we explore the influence of this assumption on the results.

As shown in Fig. 3 and previously discussed, the low $\mathrm{N}_{2}$ binding energy implies that $\mathrm{N}_{2}$ molecules remain in the gas phase in a large region where $\mathrm{CO}$ is frozen-out onto the

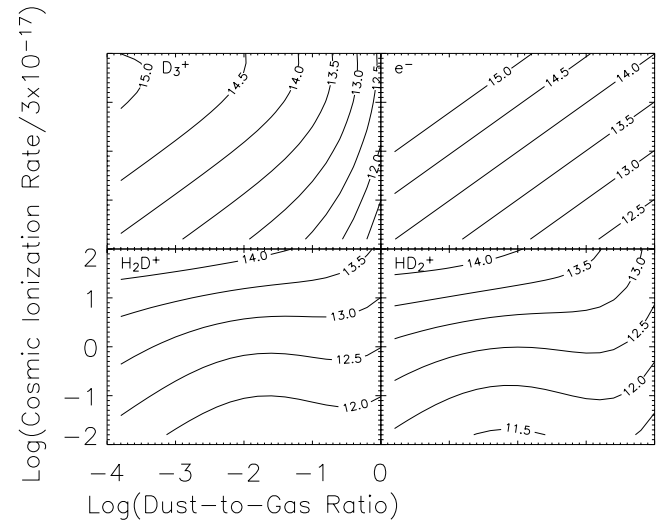

Fig. 10. Logarithm of the column density averaged over the disk surface of $\mathrm{D}_{3}^{+}$(upper left panel), $\mathrm{H}_{2} \mathrm{D}^{+}$(lower left panel), $\mathrm{HD}_{2}^{+}$(lower right panel) and electrons (upper right panel). These plots have been obtained assuming that the $\mathrm{N}_{2}$ binding energy is equal to that of $\mathrm{CO}$.

grains. It is therefore clear that the presence or not of $\mathrm{N}_{2}$ in the gas phase will have a strong impact on the modeling results.

Figure 10 shows the column densities of $\mathrm{H}_{3}^{+}, \mathrm{H}_{2} \mathrm{D}^{+}$, $\mathrm{HD}_{2}^{+}$and $\mathrm{e}^{-}$as function of the dust-to-gas ratio and the cosmic ionization ratio, similarly to Fig. 5. The comparison of the two figures speaks for itself. If $\mathrm{N}_{2}$ has a binding energy comparable to that of $\mathrm{CO}$ (Fig. 10), $\mathrm{D}_{3}^{+}$is the positive charge carrier across most of the outer disk, in the standard case (dust-to-dust ratio equal to 0.01 , and cosmic ray ionization rate equal to $3 \times 10^{-17} \mathrm{~s}^{-1}$ ), and $\mathrm{H}_{2} \mathrm{D}^{+}$and $\mathrm{HD}_{2}^{+}$are 30 times less abundant. On the contrary, if $\mathrm{N}_{2}$ has a reduced binding energy (Fig. 5) the positive charge is almost equally shared between $\mathrm{D}_{3}^{+}, \mathrm{H}_{2} \mathrm{D}^{+}$and $\mathrm{H}_{3}^{+}$. This is because the $\mathrm{N}_{2}$ collisions are an important destroyer of the $\mathrm{H}_{3}^{+}, \mathrm{H}_{2} \mathrm{D}^{+}$and $\mathrm{HD}_{2}^{+}$, preventing the "total conversion" of $\mathrm{H}_{3}^{+}$into $\mathrm{D}_{3}^{+}$.

If the $\mathrm{CO}$ and $\mathrm{N}_{2}$ binding energies are similar, increasing and/or decreasing the dust-to-gas ratio does not change much the $\mathrm{H}_{2} \mathrm{D}^{+}$and/or $\mathrm{HD}_{2}^{+}$column densities. In practice, increasing/decreasing the gas density with respect to the dust density does not change the $\mathrm{H}_{2} \mathrm{D}^{+}$and $\mathrm{HD}_{2}^{+}$column densities, but just increases/decreases the $\mathrm{D}_{3}^{+}$column density - the positive charge carrier. This has an important observational consequence, for the $\mathrm{H}_{2} \mathrm{D}^{+}$and/or $\mathrm{HD}_{2}^{+}$observed column densities cannot constrain the dust-to-gas ratio in a large interval. However, it is worth noticing that, in principle, the ratio of the derived average column densities can help to discriminate whether $\mathrm{N}_{2}$ is present in the gas phase or not. Finally, observations of the $\mathrm{N}_{2} \mathrm{H}^{+}$in proto-planetary disks will also help to clarify whether $\mathrm{N}_{2}$ remains in gas phase after $\mathrm{CO}$ condenses (Qi et al. 2003).

\section{Discussion}

\subsection{Simplified treatment of the chemical network}

In this paper we have introduced a simplified chemical network to compute the abundances of deuterated $\mathrm{H}_{3}^{+}$. The network treats only the four isotopes of $\mathrm{H}_{3}^{+}$, electrons, $\mathrm{H}^{+}, \mathrm{CO}$, and $\mathrm{N}_{2}$. Comparison with more extensive networks shows that the abundances of the $\{\mathrm{H}, \mathrm{D}\}_{3}^{+}$ions can be calculated 
accurately under the conditions of significant $\mathrm{CO}$ and $\mathrm{N}_{2}$ depletion. The reason for this success is that the chemistry indeed becomes very simple under these conditions. $\mathrm{CO}$ and $\mathrm{N}_{2}$ are the last heavy-elements bearing molecules with significant abundances to leave the gas phase and to freeze out on dust grains. Together with dust grains, they act as the main destroyers of $\mathrm{H}_{3}^{+}$and its isotopes. The simplified chemical network is, under these conditions, in fact complete in that it treats all important species and reactions. Under the assumption that the freeze-out of $\mathrm{CO}$ and $\mathrm{N}_{2}$ has reached its steady state, the steady state solution for this network can be derived directly, no time integration of the rate equations is necessary. The chemical network, therefore, lends itself for large parameter studies of cold gas.

\subsection{The main charge carrier in the disk midplane}

In the discovery paper of the $\mathrm{H}_{2} \mathrm{D}^{+}$line in DM Tau and TW Hya, Ceccarelli et al. (2004) had suggested that $\mathrm{H}_{2} \mathrm{D}^{+}$may be the main charge carrier under the conditions found in a disk, which would allow for a direct derivation of the degree of the column density of positive ions by observing $\mathrm{H}_{2} \mathrm{D}^{+}$. With an $\mathrm{H}_{2}$ column density derived from dust continuum observations, this number directly leads to the degree of ionization, a much sought-after property of disk gas because of its importance for the magneto-rotational instability, the likely driver of disk turbulence. This conclusion has to be softened in the light of the present results. Our calculations show that $\mathrm{H}_{2} \mathrm{D}^{+}, \mathrm{HD}_{2}^{+}, \mathrm{D}_{3}^{+}$ and $\mathrm{H}_{3}^{+}$all carry similar amounts of charge, indicating that the observed column densities of $\mathrm{H}_{2} \mathrm{D}^{+}$and $\mathrm{HD}_{2}^{+}$provide both an order-of-magnitude estimate of the degree of ionization, and a lower limit. It was shown earlier (Walmsley et al. 2004) that complete depletion in protostellar cores generally leaves $\mathrm{H}^{+}$as the main charge carrier. This trend, however, clearly ends at the densities found in disks: $\mathrm{H}_{3}^{+}$and its isotopes contribute equally or dominate the charge balance.

In this context, the binding energy of $\mathrm{N}_{2}$ is of critical importance. If $\mathrm{N}_{2}$ freezes out at the same moment as $\mathrm{CO}$, then the disk midplane are indeed fully depleted of heavy element bearing molecules. Then, there is no important destruction route for $\mathrm{D}_{3}^{+}$anymore, and the abundance of $\mathrm{D}_{3}^{+}$sores to dominate the charge balance. If $\mathrm{N}_{2}$ indeed stays in the gas longer than $\mathrm{CO}$, as suggested by observations in a proto-planetary disk (Qi et al. 2003), $\mathrm{H}_{2} \mathrm{D}^{+}$and $\mathrm{HD}_{2}^{+}$are produced in significant abundances and do provide a measure of the degree of ionization.

\section{3. $\mathrm{H}_{2} \mathrm{D}^{+}$and $H D_{2}^{+}$as tracers of cold gas}

One major question we aim to answer with the current study is: under what conditions are the observable lines of $\mathrm{H}_{2} \mathrm{D}^{+}$ and $\mathrm{HD}_{2}^{+}$tracers of the gas mass in the disk midplane? For these molecules to be a tracer, the observed line intensities must be a strong function of the dust-to-gas ratio. As we have seen in Figs. 5 and 10, the answer to this question depends on the behavior of $\mathrm{N}_{2}$. If $\mathrm{N}_{2}$ freezes-out at lower temperatures than $\mathrm{CO}-$ as supported by the observations in a proto-planetary disk (Qi et al. 2003) -, then the observed line intensities do depend strongly on the dust-to-gas ratio and can be used to measure it. However, under the conditions of complete depletion, i.e. if also $\mathrm{N}_{2}$ is completely removed from the midplane gas, the line intensities become insensitive to the dust-to-gas ratio.

Fortunately, observations of $\mathrm{N}_{2} \mathrm{H}^{+}$in disks can discriminate what is the reality, namely whether in the disk midplane $\mathrm{CO}$ and $\mathrm{N}_{2}$ disappear simultaneously from the gas phase or not, and, therefore, whether $\mathrm{H}_{2} \mathrm{D}^{+}$and $\mathrm{HD}_{2}^{+}$are the main positive charge carriers or $\mathrm{D}_{3}^{+}$is. In the first case, observations of $\mathrm{H}_{2} \mathrm{D}^{+}$ and $\mathrm{HD}_{2}^{+}$will give a measure of the dust-to-gas ratio, once the cosmic ionization rate is known. This also implies that $\mathrm{H}_{2} \mathrm{D}^{+}$ and $\mathrm{HD}_{2}^{+}$measure the ionization degree in the disk midplane too, as assumed by Ceccarelli et al. (2004). Once again, we emphasize that the available observations (Qi et al. 2003) support this case.

\subsection{Dust coagulation}

An important result of this study is the dependence of the chemical structure of the disk midplane on the average dust grain size. Figure 9 shows that the line intensities of the $\mathrm{o}-\mathrm{H}_{2} \mathrm{D}^{+}$and $\mathrm{p}-\mathrm{HD}_{2}^{+}$are strongly affected by the average grain sizes, if they are larger than $0.1 \mu \mathrm{m}$ radius. In practice, if small dust grains are significantly depleted in the midplane, the line intensities decrease dramatically, because $\mathrm{CO}$ and $\mathrm{N}_{2}$ do not freeze-out efficiently on the reduced grain surfaces (see the discussion in Sect. 3.2), and no substantial molecular deuteration takes place. The detection of the $\mathrm{o}-\mathrm{H}_{2} \mathrm{D}^{+}$and $\mathrm{p}-\mathrm{HD}_{2}^{+}$at the level of the current instrumental sensitivity is, therefore an indication that enough grain surface is still available to account for the molecular depletion. Two scenarios are possible for this. First, coagulation might not be efficient or aggregate destruction is efficient in keeping the number density of small grains at reasonable values. This is in fact in agreement with recent results of grain coagulation which suggest that coagulation at maximum efficiency is inconsistent with the observed opacities and shapes of flaring circumstellar disks (Dullemond \& Dominik 2005). Another possibility is that the depletion of $\mathrm{CO}$ and $\mathrm{N}_{2}$ is very efficient in the beginning, before coagulation happens. Efficient coagulation could then form large bodies that bury the ice inside and protect it from cosmic ray desorption. These considerations show that detailed models of coagulation, vertical mixing and freeze-out in combination with observations of $\mathrm{H}_{3}^{+}$isotopes can be useful diagnostics of grain coagulation.

\section{Conclusions}

We have computed the chemical structure of the midplane of disks surrounding solar type protostars. We have described the derived abundances and the resulting line intensities of the deuterated forms of $\mathrm{H}_{3}^{+}$in a standard case, and for a wide range of values of the dust-to-gas ratio and cosmic ray ionization rate. We have also discussed in detail the influence of the average grain sizes on the results. Finally, we have reported values also for different stellar luminosities, disk masses and radii, 
and discussed in detail the case of the $\mathrm{N}_{2}$ freezing onto the grain mantles simultaneously with $\mathrm{CO}$. Our main conclusions are:

$-\mathrm{H}_{3}^{+}$deuteration is significant in the midplane of protoplanetary disks around solar type protostars. In our standard case, the positive charge is carried by $\mathrm{H}_{3}^{+}$in a large zone of the disk midplane, except at the very outer radii, larger than about $300 \mathrm{AU}$, where $\mathrm{D}_{3}^{+}$takes over. $\mathrm{H}_{2} \mathrm{D}^{+}$is the most abundant $\mathrm{H}_{3}^{+}$isotope across most of the disk, at radii less than about $300 \mathrm{AU}$.

- Contrary to what was earlier assumed, $\mathrm{H}_{2} \mathrm{D}^{+}$is not the dominating positive ion in the standard case. $\mathrm{H}_{3}^{+}$and its isotopes are equally important. With increasing depletion of $\mathrm{CO}$ and $\mathrm{N}_{2}, \mathrm{D}_{3}^{+}$becomes more abundant, eventually dominating as the positive charge carrier. Also, $\mathrm{H}^{+}$is only of minor importance. While at the pre-stellar-core densities, lower than $10^{7} \mathrm{~cm}^{-3}, \mathrm{H}^{+}$dominates (Roberts et al. 2003, 2004; Walmsely et al. 2004), in the disk midplane this is no longer the case.

- The midplane chemical structure and the predicted line intensities of the $\mathrm{H}_{2} \mathrm{D}^{+}$and $\mathrm{HD}_{2}^{+}$are a strong function of the local cosmic ray ionization rate, which regulates the overall ionization degree, and the $\mathrm{CO}$ and $\mathrm{N}_{2}$ depletion across the disk.

- The chemical structure and line intensities are also sensitive to the dust-to-gas ratio as long as depletion of heavyelement bearing molecules is not complete. This happens if the $\mathrm{N}_{2}$ binding energy is lower than the $\mathrm{CO}$ binding energy, as observed in pre-stellar-cores and possibly in proto-stellar disks. If, on the contrary, $\mathrm{N}_{2}$ freezes-out simultaneously with $\mathrm{CO}$, as laboratory experiments would rather suggest, since the major charge carrier is $\mathrm{D}_{3}^{+}$, increasing/decreasing the dust-to-gas ratio does not change appreciably the $\mathrm{H}_{2} \mathrm{D}^{+}$and $\mathrm{HD}_{2}$ column densities and/or line intensities.

- The grain size has strong influence on the line strength expected for $\mathrm{H}_{2} \mathrm{D}^{+}$and $\mathrm{D}_{2} \mathrm{H}^{+}$. Small grains accelerate recombination and reduce the abundances of all positive ions, and therefore decrease the degree of ionization.

This article focuses entirely on theoretical predictions. A forthcoming paper (Dominik et al., in preparation) will analyze in detail the case of DM Tau, where the $\mathrm{o}-\mathrm{H}_{2} \mathrm{D}^{+}$line at $372 \mathrm{GHz}$ has been detected, applying the model here developed to a practical case. Likely, with the advent of ALMA, with its great sensitivity and spatial resolution, observations of both the $\mathrm{o}-\mathrm{H}_{2} \mathrm{D}^{+}$at $372 \mathrm{GHz}$ and $\mathrm{p}-\mathrm{HD}_{2}^{+}$at $691 \mathrm{GHz}$ will be possible on a routine/systematic base and with spatial resolution. Those observations promise to be very fruitful, and to bring unique information on the physical status of the midplane of the disks surrounding solar type protostars, likely similar to the progenitor of our own Solar System. Besides, observations of $\mathrm{p}-\mathrm{H}_{2} \mathrm{D}^{+}$ and o- $\mathrm{HD}_{2}^{+}$by out-of-the-atmosphere instruments will hopefully measure the actual ortho-to-para ratio of these species. Finally, $\mathrm{D}_{3}^{+}$would be likely the best diagnostics for the gas mass and ionization in low mass disks, but the only possibility to observe it is by absorption at $\sim 5 \mu$ m (Ramanlal \& Tennyson 2004; Flower et al. 2004). This requires observations towards disks almost edge-on, but not completely, for the absorption is against the inner dust continuum, which must therefore be detectable. Such disks are hard to find, but new observations by, for example, SPITZER may well discover such sources, and these absorption observations may be possible in the future.

Acknowledgements. It is a pleasure to thank Paola Caselli, Charlotte Vastel and Malcolm Walmsley for comments on the manuscript, and Kees Dullemond for his collaboration on constructing the disk model. We also wish to thank an anonymous referee for careful reading the manuscript. We acknowledge Travel support through the Dutch/French van Gogh program, project VGP 78-387.

\section{References}

Bacmann, A., Lefloch, B., Ceccarelli, C., et al. 2002, A\&A, 389, L6 Balbus, S. A., \& Hawley, J. F. 1998, Rev. Mod. Phys., 70, 1

Bergin, E. A., Langer, W. D., \& Goldsmith, P. F. 1995, ApJ, 441, 222 Bergin, E. A., \& Langer, W. D. 1997, ApJ, 486, 316

Bergin, E. A., Kaufman, M. J., Melnick, G. J., Snell, R. L., \& Howe, J. E. 2002, ApJ, 582, 830

Bergin, E. A., Alves, J., Huard, T., \& Lada, C. J. 2002, ApJ, 570, L101

Burke, J. R., \& Hollenbach, D. J. 1983, ApJ, 265, 223

Caselli, P., Walmsley, M., Tafall, M., et al. 1999, ApJ, 523, L165

Caselli, P., van der Tak, F. F. S., Ceccarelli, C., \& Bacmann, A. 2003, A\&A, 403, L37

Ceccarelli, C. 2004, in The Dense Interstellar Medium in Galaxies, ed. S. Pfalzner, C. Kramer, C. Straubmeier, \& A. Heithausen, Springer Proceedings in Physics

Ceccarelli, C., Dominik, C., Lefloch, B., Caselli, P., \& Caux, E. 2004, ApJ, 607, L651

Ceccarelli, C., Maret, S., Tielens, X., Castets, A., \& Caux, E. 2003, A\&A, 410, 587

Collings, M. P., Dever, J. W., Fraser, H. J., McCoustra, M. R. S., \& Williams, D. A. 2003, ApJ, 583, 1058

Crapsi, A., Caselli, P., Walmsley, C. M., et al. 2005, ApJ, 619, 379

Draine, B. T., \& Sutin, B. 1987, ApJ, 320, 803

Dominik, C., Dullemond, C. P., Waters, L. B. F. M., \& Walch, S. 2003, A\&A, 398, 607

Dullemond, C. P., Dominik, C., \& Natta, A. 2001, ApJ, 560, 957

Dullemond, C. P., \& Dominik, C. 2004, A\&A, 417, 159

Dullemond, C. P., \& Dominik, C. 2005, A\&A, 434, 971

Flower, D., Pineau des Forets, G., \& Walmsley, M. 2004, A\&A, 427, 887

Fraser, H. J., Collings, M. P., Dever, J. W., \& McCoustra, M. R. S. 2004, MNRAS, 353, 59

Frerking, M. A., Langer, W. D., \& Wilson, R. W. 1982, ApJ, 262, 590

Gammie, C. F. 1996, ApJ, 457, 355

Hasegawa, T. I., \& Herbst, E. 1993, MNRAS, 263, 589

McCall, B. J., Huneycutt, A. J., Saykally, R. J., et al. 2003, Nature, 422,500

Oberg, K. I., van Broekhuizen, F., Fraser, H. J., et al. 2005, ApJ, 621, L330

Pagani, L., Pardo, J.-R., Apponi, A. J., Bacmann, A., \& Cabrit, S. 2004, A\&A, 249, 181

Qi, C., Kessler, J. E., Koerner, D. W., Sargent, A. I., \& Blake, G. A. 2003, ApJ, 597, 986

Ramanlal, J., \& Tennyson, J. 2004, MNRAS, 354, 161

Roberts, H., Herbst, E., \& Millar, T. J. 2003, ApJ, 591, L41

Roberts, H., Herbst, E., \& Millar, T. J. 2004, A\&A, 424, 905

Semenov, D., Wiebe, D., \& Henning, Th. 2004, A\&A, 417, 93

Tafalla, M., Meyers, P., Caselli, P., Walmsley, M., \& Comito, C. 2002, ApJ, 569, 815

Tafalla, M., Meyers, P., Caselli, P., \& Walmsley, M. 2004, A\&A, 416, 191

Walmsley, M., Flower, D., \& Pineau des Forets, G. 2004, A\&A, 418, 1035 